\title{
Regulación emocional y memoria de trabajo en el desempeño académico
}

\section{Emotion regulation and working memory in academic performance}

\section{Regulação emocional e memória de trabalho no desempenho acadêmico}

\author{
María Laura Andrés ${ }^{1}$, ORCID 0000-0003-4398-2043 \\ Santiago Vernucci ${ }^{2}$, ORCID 0000-0003-1595-3106 \\ Ana García Coni ${ }^{3}$, ORCID 0000-0002-6304-7880 \\ María M. Richard's ${ }^{4}$, ORCID 0000-0002-9425-193X \\ Mariela Luz Amazzini ${ }^{5}$, ORCID 0000-0003-0138-7406 \\ Rosaura Paradiso ${ }^{6}$, ORCID 0000-0002-4845-1869 \\ 1234 Instituto de Psicología Básica, Aplicada y Tecnología IPSIBAT (UNMDP-CONICET). \\ Argentina \\ ${ }^{56}$ Facultad de Psicología (UNMDP). Argentina
}

\begin{abstract}
Resumen: El objetivo de este trabajo fue analizar la relación de las estrategias de regulación emocional reevaluación cognitiva aplicada a tareas escolares para el hogar (RC-TEH) y manejo emocional aplicado a tareas escolares para el hogar (ME-TEH) sobre el desempeño académico (DA) controlando dificultades de memoria de trabajo (dif-MT) en niños que finalizaban la Educación Primaria. Se administró un cuestionario de autoinforme para evaluar la RC-TEH y el ME-TEH a 119 de niños de cuarto, quinto y sexto grado (9 a 11 años de edad) y dos cuestionarios a sus maestros para evaluar su DA y dificultades de MT. Los resultados mostraron que el ME-TEH se asoció con las dimensiones éxito, productividad académica, matemáticas y lectura del DA, incluso cuando se controlaron dif-MT. No se observaron asociaciones significativas de la RC-TEH con el DA, ni de ninguna de las estrategias sobre el control del impulso en situaciones académicas. Se espera que los resultados profundicen en el conocimiento del rol de la regulación emocional en el DA y contribuyan así al contenido de programas de promoción de la regulación emocional diseñados para contextos escolares.
\end{abstract}

Palabras clave: regulación emocional; memoria de trabajo; desempeño académico; niños; docentes

Abstract: The objective of this work was to analyze the relationship between emotional regulation strategies, cognitive reappraisal applied to home school tasks (CR-HST) and emotional management applied to home school tasks (EM-HST) on academic performance (AP) controlling working memory difficulties (WM-diff) in children who finished Primary Education. A self-report questionnaire to assess CR-HST and EM-HST was administered to 119 fourth, fifth and sixth grade children ( 9 to 11 years old) and two questionnaires to their teachers to assess their AP and WM difficulties. The results showed that EM-HST was associated with the success, academic productivity, mathematics and reading dimensions of AP, even when WM-diff was controlled. There were no significant associations between CR-HST and AP, or any of the strategies on impulse control in academic situations. The results are expected to deepen the understanding of the role of emotional regulation in AP and thus contribute to the content of programs for promoting emotional regulation designed for school contexts.

Keywords: emotion regulation; working memory; academic performance; children; teachers 
Resumo: O objetivo deste trabalho foi analisar a relação entre as estratégias de regulação emocional de reavaliação cognitiva aplicada ao dever de casa (RC-DDC) e a gestão emocional aplicada ao dever de casa (GE-DDE) sobre o desempenho acadêmico (DA) controlando as dificuldades de memória de trabalho (dif-MT) em crianças que terminam o Ensino Fundamental. Foi administrado um questionário de auto-relato para avaliar RC-DDC e GE-DDE a 119 crianças de quarto, quinto e sexto grau (9-11 anos), e dois questionários foram administrados a seus professores para avaliar suas dificuldades de DA e MT. Os resultados mostraram que a RC-DDC estava associada ao sucesso, produtividade acadêmica, matemática e leitura da DA, mesmo quando se controla a dif-MT. Não foram observadas associações significativas para RC-DDC com DA, nem para nenhuma das estratégias de controle de impulsos em situações acadêmicas. Espera-se que os resultados aprofundem a compreensão do papel da regulação emocional na DA e assim contribuam para o conteúdo de programas que promovam a regulação emocional projetada para ambientes escolares.

Palavras-chave: regulação emocional; memória de trabalho; desempenho académico; crianças; professores

Recibido: 15/10/2019

Aceptado: 14/08/2020

Cómo citar:

Andrés, M.L., Vernucci, S., García Coni, A., Richard's, M.M., Amazzini, M.L., \& Paradiso, R. (2020). Regulación emocional y memoria de trabajo en el desempeño académico. Ciencias Psicológicas, 14(2), e2284. doi: https://doi.org/10.22235/cp.v14i2.2284

Correspondencia: María Laura Andrés, marialauraandres@gmail.com; Santiago Vernucci, santiago.vernucci@gmail.com; Ana García Coni, anagconi@gmail.com; María M. Richard's, mariamartarichards@gmail.com. Instituto de Psicología Básica, Aplicada y Tecnología IPSIBAT (UNMDPCONICET), Funes 3250 Cuerpo V Nivel III Mar del Plata CP: 7600. (Buenos Aires, Argentina). Mariela Luz Amazzini, luzmarielaammazzini@gmail.com; Rosaura Paradiso, rosauraparadiso@gmail.com. Facultad de Psicología (UNMDP), Funes 3250, Cuerpo V, Nivel III, Mar del Plata CP 7600. (Buenos Aires, Argentina).

\section{Introducción}

El desempeño académico (DA) se define como el nivel de logro de metas educativas o de conocimientos alcanzado en un área específica (Steinmayr, Meibner, Weidinger, \& Wirthwein, 2015). El DA compromete no solamente la inserción laboral futura de los niños (Parsons \& Bynner, 2005) sino también su bienestar psicológico presente (Bennett, Brown, Boyle, Racine \& Offord, 2003). Debido a su importancia, se han analizado los factores socioculturales, familiares e individuales que contribuyen a un DA exitoso (e.g. Hattie, 2009). Uno de los factores individuales de carácter afectivo que ha comenzado a cobrar valor es la regulación de las emociones (Martin \& Ochsner, 2016).

La regulación emocional se define como aquellos procesos mediante los cuales los individuos influyen en sus emociones, en cuándo y cómo las experimentan y expresan (Gross, 2014). En relación al DA, numerosos procesos de regulación emocional han sido estudiados respecto de su capacidad predictiva (Andrés et al., 2017). Recientemente se ha prestado particular atención a las estrategias de regulación de la emoción de tareas escolares para el hogar, es decir, aquellas estrategias de regulación emocional aplicadas específicamente a las emociones -normalmente de displacer- que suelen generar las tareas escolares para el hogar $(\mathrm{Xu}, \mathrm{Fan} \& \mathrm{Du}$, 2016). Dichas tareas se definen como actividades asignadas por los docentes a los alumnos para que sean realizadas fuera del horario escolar con diferentes propósitos, entre los que se 
encuentran el repaso de material presentado en clase, la preparación de nuevos materiales, la transferencia de habilidades a otros dominios y/o la integración de diferentes conceptos (Cooper, Robinson \& Patall, 2006). Si bien las tareas escolares poseen una influencia positiva sobre el DA (Bas, Senturk, \& Cigerci, 2017; Cooper et al., 2006; Maltese, Tai \& Fan, 2012), es sabido que pueden generar emociones displacenteras que en algunos casos impiden la finalización de las mismas (Xu, 2016); de modo que la capacidad de regular estas emociones debería poseer impacto sobre el DA.

Uno de los modelos de regulación emocional con mayor soporte empírico es el modelo procesual de Gross (2014), que destaca la importancia del cambio cognitivo como componente efectivo para la disminuir las emociones negativas. El cambio cognitivo es un conjunto que incluye estrategias de "actuación temprana" que se distinguen de las de "actuación tardía", algunas de las cuales han mostrado poca efectividad (e.g. supresión de la expresión emocional) (Gross, 2002). El cambio cognitivo significa cambiar las evaluaciones sobre una situación para alterar su impacto emocional, tanto sea mediante la modificación de los pensamientos respecto de la situación o de las propias capacidades para manejar sus demandas (Gross \& Thompson, 2007). Dentro de este componente se destacan dos estrategias: la reevaluación cognitiva implica cambiar el significado de una situación para alterar su impacto emocional; mientras que el manejo emocional implica modificar los pensamientos sobre las propias capacidades para impactar en la emoción (Gross \& Thompson, 2007).

En cuanto a la evidencia empírica específica de la relación de estrategias con el DA, una revisión reciente de estudios (Andrés et al., 2017) indicó que la implementación de la reevaluación cognitiva mejoró el rendimiento en matemáticas (Jamieson et al., 2010; Johns, Inzlicht \& Schmader 2008) y el recuerdo para información educativa tanto en adultos (Leroy, Grégoire, Magen, Gross \& Mikolajczak, 2012) como en niños de escolaridad primaria (Davis \& Levine, 2013). Sin embargo, su implementación no mejoró el rendimiento en comprensión lectora (Jamieson et al., 2010). En cuanto a la reevaluación cognitiva aplicada específicamente a tareas escolares para el hogar (RC-TEH), su frecuencia de uso autoinformada se asoció positivamente con la tendencia a completar tareas en matemáticas, pero no con pruebas estandarizadas en dicho dominio (Xu et al., 2015, 2016). Respecto del manejo emocional, distintos trabajos hallaron que su implementación repercutió positivamente en el rendimiento en matemáticas (Jamieson et al., 2010; Johns et al., 2008) y en el recuerdo de material educativo (Leroy et al., 2012); y en cuanto al manejo emocional aplicado específicamente a tareas escolares para el hogar (ME-TEH), Xu et al. $(2015,2016)$ observaron asociaciones positivas con pruebas estandarizadas de matemáticas.

De los estudios anteriores, sólo uno incluyó niños de escolaridad primaria (i.e. Davis \& Levine, 2013), sin analizar el rol de la regulación de la emoción de tareas escolares. Por tanto, realizar este análisis representaría un aporte. Por un lado, permitiría ampliar el conocimiento acerca de la regulación de la emoción de tareas escolares; y por otro, permitiría considerar los importantes cambios a nivel cognitivo, emocional y académico que se producen en niños que se encuentran en los últimos años de su escolaridad primaria. Durante este período se desarrollan y maduran las funciones ejecutivas, aumentan las demandas sociales y académicas; por lo que la regulación emocional resulta más demandada. En este sentido, las funciones ejecutivas se ponen al servicio tanto de las demandas socioemocionales como académicas (Center on the Developing Child at Harvard University, 2011; Diamond, 2013; St Clair, Thompson, \& Gathercole, 2006).

Al considerar lo anterior, cobra valor analizar el papel de las funciones ejecutivas en la asociación de la regulación emocional con el DA, especialmente de la memoria de trabajo. La memoria de trabajo constituye un sistema complejo de capacidad limitada encargado de almacenar y procesar información de manera simultánea (Baddeley, 2012) y resulta crucial en un DA exitoso (Alloway, T.P. \& Alloway, R.G., 2010). Por ejemplo, en la comprensión lectora la memoria de trabajo almacena las oraciones leídas para que puedan integrarse entre sí, a la vez 
que activa información de la memoria a largo plazo para que pueda integrarse con lo anterior, favoreciendo así la construcción de una representación coherente del texto (Cain, Oakhill \& Bryant, 2004). En las operaciones de cálculo, la memoria de trabajo permite mantener activas combinaciones aritméticas provenientes tanto de la memoria a largo plazo como del conteo, mientras se atiende a las demandas de reagrupación que la operación requiera (Bull \& Lee, 2014; Raghubar, Barnes \& Hecht, 2010). Diversos estudios han señalado el rol predictor de la memoria de trabajo en el DA, (e.g. Alloway, T.P. \& Alloway, R.G., 2010), e incluso se han observado asociaciones cuando se utilizan como medida escalas de reporte para su evaluación (e.g. Vernucci et al., 2020).

La memoria de trabajo juega un rol clave en la autorregulación general (Hofmann, Schmeichel, \& Baddeley, 2012), y específicamente en la regulación emocional, ya que permite, por ejemplo, reemplazar las evaluaciones iniciales de un evento emocional con evaluaciones secundarias con menor valencia emocional (Pe, Raes \& Kuppens, 2013), un proceso clave para la reevaluación cognitiva y el manejo emocional. Existe evidencia que muestra la importancia de la memoria de trabajo en la reevaluación cognitiva tanto en adultos (e.g. McRae, Jacobs, Ray, John \& Gross, 2012; Pe et al., 2013, 2015) como en niños (Andrés, Castañeircas, Stelzer, Canet Juric \& Introzzi, 2016). De modo que analizar la relación de la regulación emocional con el DA controlando el peso de la memoria de trabajo - particularmente de las dificultades de memoria de trabajo (dif-MT) observadas por los docentes- sería importante para conocer la capacidad predictiva de la regulación emocional con mayor precisión. El único antecedente que es posible mencionar en este aspecto es el estudio de LaFavor (2012), quien encontró una contribución explicativa única de la tolerancia al distrés - una habilidad de regulación emocional que consiste en resistir estados emocionales negativos al servicio de un objetivo- sobre el DA cuando se controlan las funciones ejecutivas en niños en situación de vulnerabilidad social que viven en la calle. Analizar estas relaciones en otros contextos poblacionales y sobre procesos de regulación emocional más vinculados al DA tales como las estrategias de regulación de la emoción de tareas escolares para el hogar resultaría de valor para complementar el conocimiento disponible.

Al tener en cuenta lo anterior, el objetivo de este trabajo es analizar la relación de la regulación emocional -particularmente de las estrategias de regulación RC-TEH y ME-TEHsobre el DA controlando el peso de las dif-MT, en un grupo de niños que se encuentran en los últimos años de la escolaridad primaria. Se espera que aquellos niños que informen mayor uso de la RC-TEH y el ME-TEH sean evaluados por sus docentes con mejor DA, incluso cuando se controlen las dif-MT informadas por estos mismos.

\section{Materiales y Método}

\section{Diseño y Participantes}

Se trata de un estudio ex post facto o correlacional según la clasificación de Montero y León (2007) en el que fueron evaluados de forma intencional no probabilística 119 niños de 9 a 11 años de edad $(M=10.85 ; D E=0.85)$ pertenecientes a cuarto $(n=37 ; M=9.86 ; D E=0.31)$, quinto $(n=47 ; M=10.84 ; D E=0.25)$, y sexto grado $(n=35 ; M=11.92 ; D E=0.29)$ de la Educación Primaria de ambos sexos (55.5\% de mujeres y $44.5 \%$ de varones) de institución escolar de gestión privada de la ciudad de Mar del Plata (Buenos Aires, Argentina). Las familias de los niños pertenecían en su mayoría a un estatus social alto y medio alto (68.1\%) y el resto se distribuyó entre medio y medio bajo. 


\section{Instrumentos}

- Estrategias de regulación de la emoción aplicadas a tareas escolares para el hogar: $R C$-TEH y ME-TEH. Se administró la Escala de Regulación Emocional de Tareas Escolares para el Hogar HERS (Xu, Fan \& Du, 2015). Se compone de seis ítems que se responden en escala Likert de cinco opciones desde "nunca" (1) hasta "siempre" (5). La subescala RC-TEH evalúa los esfuerzos para resignificar el displacer de las tareas escolares para el hogar en términos menos emocionales, y se compone de tres ítems: "Pienso que hacer las tareas tiene su lado bueno" (ítem 1), "Pienso que puedo aprender algo bueno de la situación" (ítem 4), y "Pienso que no es tan terrible tener que hacerlas" (ítem 6). La subescala ME-TEH evalúa los esfuerzos de los alumnos por cambiar la forma de pensar sobre la propia capacidad de manejar las demandas emocionales de las tareas escolares, y se compone de tres ítems: "Pienso que no es tan importante si me equivoco" (ítem 2), "Pienso que tengo que estar tranquilo" (ítem 3), y "Pienso que soy capaz de hacer bien las tareas" (ítem 5). Las propiedades psicométricas reportadas por los autores del instrumento original son adecuadas. El análisis factorial confirmatorio mostró la conformación de dos factores de acuerdo con los ítems componentes de cada subescala (CFI=0.98; SRMR=0.02; RMSEA=0.06 [90\% CI =0.048- 0.091]). En cuanto a los análisis de confiabilidad, los valores alpha de Cronbach fueron adecuados $(\alpha=.83$ para ME-TEH y $\alpha=.87$ para RC-TEH). El análisis de validez concurrente y predictiva mostró asociaciones de las subescalas con orientación al dominio y desempeño, cantidad de tareas completadas, estrategias de aprendizaje y desempeño en tareas escolares para el hogar informado por los docentes. Se observaron asociaciones negativas de las subescalas con orientación a la evitación y la frecuencia de tareas incompletas (Xu et al., 2015).

Para ser utilizado en nuestro medio, el instrumento fue traducido y adaptado. Se realizó una traducción de los ítems del inglés al castellano cuidando que la misma fuera funcional y no literal (Hambleton, 2001). Luego, se solicitó a psicólogos expertos en la temática y con dominio del inglés que revisaran estos ítems en cuanto a claridad semántica y gramatical, correspondencia con el dominio que se buscaba medir y adecuación al nivel educativo y evolutivo de los participantes a quienes estaba dirigido (Tornimbeni, Pérez, Olaz \& Fernández, 2004). Luego de su aplicación a la muestra bajo estudio se realizó un análisis factorial exploratorio mediante el método de Componentes Principales y rotación Oblimin (KMO=.723; Prueba de esfericidad de Barlett=91.23: $p<.001$ ). Éste indicó la conformación de dos factores que explicaron el $54.14 \%$ de la varianza, donde los ítems cargaron en los factores a los que pertenecen teóricamente, excepto el ítem 5 cuya carga se repartió parcialmente con el componente de RC. La matriz de correlaciones bivariadas mostró que el ítem 2 posee una asociación negativa con todos los ítems. Es posible que hubiera sido interpretado con una connotación negativa por parte de los niños y no como una forma de manejo de las demandas emocionales de la situación, por lo que se excluyó de la conformación de las subescalas. El análisis de fiabilidad del instrumento resultó entre moderado y bajo ( $\alpha$ total=.64; ME-TEH: $\alpha=.48$; RC-TEH: $\alpha=.41$ ).

- Desempeño académico. Se administró la Escala de Clasificación del Desempeño Académico APRS (DuPaul, Rapport \& Perriello, 1991) que refleja las percepciones docentes del desempeño y habilidades académicas de los niños en el aula. Se compone de 19 ítems que los docentes responden utilizando una escala tipo Likert de cinco opciones de respuesta, desde 1 (nunca o pobre) hasta 5 (muy a menudo o excelente) que se agrupan en tres subescalas (algunos ítems son compartidos por dos subescalas) que representan tres dimensiones del DA: Éxito académico (7 ítems), se refiere al logro académico obtenido, tales como la calidad de las producciones y la habilidad y velocidad para aprender y recuperar nuevo material (e.g. "¿Cómo es en términos generales la calidad de sus trabajos escolares?"). Control del impulso (3 ítems), alude a la regulación del comportamiento en clase y en situaciones académicas (e.g. "¿Con cuánta frecuencia este niño completa las tareas de una manera descuidada y apresurada?"). 
Productividad académica (12 ítems), hace referencia a aquellos comportamientos importantes para el logro del éxito académico tales como completar las tareas, seguir instrucciones y trabajar de forma independiente y en tiempo (e.g. “¿Con cuánta frecuencia el niño sigue de modo preciso las instrucciones del docente que se dan a todo el grupo?"). Entre los ítems que componen las subescalas algunos están referidos a habilidades académicas específicas, como matemática y lectura (e.g. "Estime el porcentaje de tareas correctamente realizadas en matemática -preguntas, ejercicios, etc.-”; “¿Cuál es la calidad de las habilidades de lectura de este niño?”). En cuanto a las propiedades psicométricas del instrumento, los análisis realizados por los autores de la escala indican que son adecuadas. El análisis factorial exploratorio mostró la conformación de tres factores correspondientes a las tres subescalas que explicaron el $68 \%$ de la varianza. La consistencia interna de la escala resultó adecuada tanto para la totalidad de la escala $(\alpha=.95)$ como para cada una de las subescalas (desde $\alpha=.72$ hasta $\alpha=.94$ ), y el coeficiente test-retest fue alto tanto para la escala total $(r=.95)$ como las subescalas (desde $r=.88$ hasta $r=.93$ ). Las relaciones de la escala con variables teóricamente relacionadas tales como eficiencia académica, indicadores comportamentales y pruebas estandarizadas de habilidades académicas fueron significativas en la mayoría de los casos (24 de los 28 coeficientes de correlación fueron significativos) (DuPaul et al., 1991).

Para ser aplicado a la muestra bajo estudio se siguió el mismo procedimiento que el realizado con la escala HERS (ver descripción anterior). El análisis factorial exploratorio mediante el método de Componentes Principales y rotación Varimax (KMO=.90; Prueba de esfericidad de Barlett=2739.49; $p<.001)$ mostró la conformación de tres factores que explicaron el $76.33 \%$ de la varianza, donde 13 de los 19 ítems cargaron en los factores a los que pertenecen teóricamente. El resto mostró cargas parcialmente repartidas con otro factor. El análisis de fiabilidad arrojó valores alpha adecuados ( $\alpha=.96$ para todo el instrumento; $\alpha=.94$ productividad académica; $\alpha=.94$ éxito académico y $\alpha=.72$ control del impulso).

- Dificultades de memoria de trabajo. Se aplicó la versión traducida al español de la Escala de Observación de Memoria de Trabajo WMRS (Alloway, Gathercole \& Kirkwood, 2008) adaptada a nuestro medio local (Vernucci et al., 2020). Está conformada por 20 descripciones breves de problemas de comportamiento asociados a un bajo nivel de funcionamiento de MT, y permite discernir entre niños con escasas habilidades de MT y niños con habilidades promedio. El docente puntúa cuán típico es cada comportamiento en una escala tipo Likert de cuatro opciones de respuesta desde 0 (para nada típico) hasta 3 (muy típico). Ejemplos de ítems son: "Levanta la mano para responder una pregunta, pero olvida lo que iba a decir cuando le preguntan"; "Necesita recordatorios regulares sobre cada paso de una tarea escrita"; "Depende de su compañero de banco para recordar lo central de la actividad en curso". Puntuaciones más altas indican mayores dificultades de MT. El análisis de las propiedades psicométricas realizado por los autores del instrumento muestra buenos indicadores internos de confiabilidad, validez convergente con medidas tradicionales de MT como el Test AWMA (Automated Working Memory Assessment; Alloway, 2007) y el WISC-IV (Weschler, 2004). La versión traducida al español y adaptada a nuestro contexto aplicada en el presente estudio, ha mostrado adecuadas propiedades psicométricas, en los $\mathrm{AFC}$ (CFI=.99; SMRM=.04; PMFI=.48), y confiabilidad $(\alpha=.97)$ (Vernucci et al., 2020). En nuestra muestra presentó un valor alpha elevado $(\alpha=.97)$.

- Estatus Social. Se administró una encuesta para conocer el estatus social de las familias de los niños. La misma indaga por el máximo nivel de escolaridad alcanzado y el tipo de ocupación del principal sostén económico. Esta información se clasifica luego mediante una escala basada en el sistema educativo nacional (Pascual, Galperín \& Bornstein, 1993) y la Escala de Prestigio Ocupacional EGO70 (Sautú, 1989). El estatus social se calculó utilizando el Índice de Hollingshead (2011) que resulta adecuado en nuestro medio (Pascual et al., 1993). 


\section{Procedimiento y consideraciones éticas}

Se realizaron reuniones con los equipos directivos de las instituciones invitadas a participar del estudio, para brindar información sobre los objetivos, instrumentos y procedimientos. Luego, se envió a los padres un documento informativo, junto con el formulario de consentimiento informado. Fueron incluidos en la muestra aquellos niños cuyos padres dieron el consentimiento y que prestaron su asentimiento al momento de realizar las evaluaciones. La administración tuvo lugar dentro del establecimiento educativo en el horario escolar habitual. La escala HERS fue completada por los mismos niños de manera grupal en el salón habitual de clase, las escalas APRS y WMRS fueron completadas por el principal docente del niño y la encuesta de estatus social fue enviada a los padres a través del cuaderno de comunicaciones. En el desarrollo del presente trabajo de investigación se respetaron los lineamientos para el comportamiento ético en las Ciencias Sociales y Humanidades dados por el Consejo Nacional de Investigaciones Científicas y Técnicas (CONICET, 2006), así como los comprendidos para las actividades destinadas a obtener conocimientos sobre procesos psicológicos en seres humanos, recomendados por la American Psychological Association (APA, 2010).

\section{Plan de análisis de los datos}

En primer lugar, se realizaron estadísticos descriptivos de las variables (media, desviación estándar, índices de asimetría y curtosis). Se evaluó cada variable respecto de la presencia de outliers univariados (valores superiores a $\pm 3 \mathrm{SD}$ ), los cuales fueron eliminados. Se analizó la normalidad de las distribuciones en base a los valores de asimetría y curtosis $( \pm 1$ es considerado aceptable). Se realizaron correlaciones bivariadas de Pearson para estimar la relación entre las diferentes variables bajo estudio. En segundo lugar, se analizó si existían diferencias en las variables en función del género, el grado escolar y estatus social de los participantes mediante un ANOVA factorial. En tercer lugar, se realizaron análisis de regresión lineal múltiple en dos bloques o modelos para analizar la relación de las variables independientes con el DA y se introdujeron aquellas variables sociodemográficas que generaron diferencias como variables de control; en el primer bloque o modelo se introdujeron las estrategias de regulación emocional y en el segundo se adicionó dif-MT. De esta manera, es posible ver el cambio en la capacidad explicativa de las estrategias cuando se introduce dif-MT. Las variables dependientes de DA fueron el éxito académico, el control del impulso y la productividad académica. Adicionalmente, se sumaron los ítems de la escala APRS que hacen referencia a las áreas de matemáticas y lectura y se analizó la capacidad predictiva de las estrategias y las dif-MT sobre ellas para conocer si existe una influencia específica y diferencial sobre estas habilidades académicas.

\section{Resultados}

\section{Análisis descriptivos y de correlación}

Los estadísticos descriptivos y las correlaciones de Pearson entre las variables se presentan en la Tabla 1. Se observa que en todos los casos los valores de asimetría y curtosis permiten suponer una distribución normal de los datos. No se observaron outliers por lo que no se eliminaron casos de la muestra. Como se observa en la Tabla 1, el ME-TEH fue la estrategia que presentó asociaciones significativas con las dimensiones del DA. Las dif-MT no se asociaron con las estrategias de regulación emocional. 
Tabla 1

Estadísticos descriptivos y correlaciones bivariadas de las variables bajo estudio

\begin{tabular}{|c|c|c|c|c|c|c|c|c|c|c|c|}
\hline & $\mathbf{M}$ & DE & Asimetría & Curtosis & 2 & 3 & 4 & 5 & 6 & 7 & 8 \\
\hline $\begin{array}{l}\text { 1.RC- } \\
\text { TEH }\end{array}$ & 10.62 & 2.76 & -.27 & -.61 & $.41^{* *}$ & .04 & .06 & .02 & .03 & .03 & -.01 \\
\hline $\begin{array}{l}\text { 2.ME- } \\
\text { TEH }\end{array}$ & 8.04 & 1.64 & -.57 & -.58 & & $.19^{*}$ & .16 & $.24^{* *}$ & $.22^{*}$ & $.20^{*}$ & -.09 \\
\hline $\begin{array}{l}\text { 3.Éxito } \\
\text { Ac. }\end{array}$ & 26.48 & 6.22 & -.17 & -.67 & & & $.75^{* *}$ & $.93^{* *}$ & $.90^{* *}$ & $.97^{* *}$ & $-77^{* *}$ \\
\hline $\begin{array}{l}\text { 4.Ctrol. } \\
\text { Im. }\end{array}$ & 11.85 & 2.19 & -.51 & .01 & & & & $.72^{* * *}$ & $.69^{* *}$ & $.73^{* * *}$ & $-64^{* *}$ \\
\hline $\begin{array}{l}\text { 5.Prod. } \\
\text { Ac. }\end{array}$ & 46.56 & 9.69 & -.58 & .00 & & & & & $.94^{* *}$ & $.93^{* *}$ & $-77^{* *}$ \\
\hline 6.Mat. & 7.45 & 2.14 & -.62 & -.29 & & & & & & $.93^{* *}$ & $-73^{* *}$ \\
\hline 7.Lectura & 11.13 & 3.03 & -.31 & -.70 & & & & & & & $-74^{* *}$ \\
\hline $\begin{array}{l}\text { 8.Dif- } \\
\text { MT }\end{array}$ & 10.06 & 10.94 & -90 & -.26 & & & & & & & $-79^{* * *}$ \\
\hline
\end{tabular}

Nota. Éxito Ac.=éxito académico; Ctrol. Im.=control del impulso; Prod. Ac.=productividad académica; Mat.=matemáticas.

$* p<.05 ; * * p<.01$

\section{Análisis de las diferencias según género, grado escolar y estatus social}

Se realizó un ANOVA factorial introduciendo como factores fijos el género, el grado escolar y el estatus social de los participantes y como variables dependientes las estrategias de regulación emocional, las dif-MT y DA. Se observó un efecto principal del grado sobre las difMT $(F(2)=6.14 ; p<.01)$ y el DA $(F(2)=5.01 ; p<.01)$. No se observaron efectos principales ni de interacción del resto de los factores y ninguno de los factores afectó a las estrategias de regulación emocional. Los estadísticos descriptivos muestran diferencias entre las medias de cada grado en dif-MT (cuarto grado: $M=6.70 ; D E=9.2$; quinto grado: $M=8.66 ; D E=9.40$; sexto grado: $M=15.51 ; D E=12.62$ ) y en $D A$ (cuarto grado: $M=90.98 ; D E=15.49$; quinto grado: $M=87.11 ; D E=14.39$; sexto grado: $M=75.62 ; D E=19.43)$.

\section{Relación de la regulación emocional y MT con DA: análisis de regresión lineal múltiple}

Se analizó el cumplimiento de los supuestos en los modelos de regresión. El estadístico de Durbin-Watson mostró en todos los casos valores entre 1.70 y 1.89 (independencia). Los gráficos de dispersión entre los residuos estandarizados y los valores predichos estandarizados no mostraron pautas de asociación (homocedasticidad). Los residuos se distribuyeron normalmente $(M=.00 ; D E=1.00$ en todos los modelos) y tanto el histograma como el gráfico de probabilidad normal mostraron una distribución cercana a la normal (normalidad). Los gráficos parciales mostraron nubes de puntos cercanas a la recta (aunque para el caso de la RC-TEH los residuos se alejan de la recta relativamente) (cumplimiento parcial del supuesto de linealidad). Finalmente, los valores de tolerancia fueron entre .80 y .99 y los de VIF entre 1.01 y 1.20 , no se observaron autovalores cercanos a 0 , todos los índices de condición fueron menores a 15 en todos los modelos y la proporción de varianza explicada para cada dimensión fue grande en un solo coeficiente por modelo (no-colinealidad).

En el primer modelo (ver Tabla 2) se introdujo el grado escolar como variable de control -debido a los resultados del ANOVA factorial- y las estrategias de regulación emocional, y en el segundo se adicionó las dif-MT como otra variable de control. De esta manera, puede observarse 
el cambio en la capacidad explicativa de ambos modelos, donde si bien la del modelo general aumenta, la de las estrategias disminuye; pero la asociación se mantiene. La mayor capacidad predictiva estuvo dada por las dif-MT. La regulación emocional se asoció únicamente mediante la estrategia ME-TEH con las dimensiones del DA productividad, matemática y lectura, marginalmente con éxito académico y no lo hizo con control del impulso.

Tabla 2

Análisis de regresión lineal múltiple: relación de las estrategias de regulación emocional y la MT con el $D A$

\begin{tabular}{|c|c|c|c|c|c|c|}
\hline & & $\begin{array}{c}\text { Éxito } \\
\text { Académico } \\
\beta \\
\end{array}$ & $\begin{array}{c}\text { Control del } \\
\text { Impulso } \\
\beta \\
\end{array}$ & $\begin{array}{c}\text { Productivida } \\
\text { d Académica } \\
\beta \\
\end{array}$ & $\begin{array}{c}\text { Matemáticas } \\
\beta \\
\end{array}$ & $\begin{array}{c}\text { Lectura } \\
\beta \\
\end{array}$ \\
\hline \multirow[t]{4}{*}{ Modelo 1} & & $\begin{array}{l}F(3)=7.48 ; \\
p<.001 \\
R^{2}=.14\end{array}$ & $\begin{array}{l}F(3)=8.08 ; \\
p<.01 ; \\
R^{2}=.08\end{array}$ & $\begin{array}{l}F(3)=6.61 ; \\
p<.001 \\
R^{2}=.12\end{array}$ & $\begin{array}{l}F(3)=6.97 ; \\
p<.001 ; \\
R^{2}=.13\end{array}$ & $\begin{array}{l}F(3)=7.09 ; \\
p<.001 \\
R^{2}=.13\end{array}$ \\
\hline & $\begin{array}{l}\text { Grado } \\
\text { Escolar }\end{array}$ & $-.35 * * *$ & $-.29 * *$ & $-.20 * *$ & $-.31 * * *$ & $-.33 * * *$ \\
\hline & RC-TEH & .04 & -.00 & -.09 & -.07 & -.06 \\
\hline & ME-TEH & $.17(p=.06)$ & .13 & $.25^{* *}$ & $.21^{*}$ & $.19^{*}$ \\
\hline \multirow[t]{4}{*}{ Modelo 2} & & $\begin{array}{l}F(4)=48.77, \\
p<.001, \\
R^{2}=.61\end{array}$ & $\begin{array}{l}F(4)=22.48 \\
p<.001 \\
R^{2}=.42\end{array}$ & $\begin{array}{l}F(4)=48.99, \\
p<.001, \\
R^{2}=.61\end{array}$ & $\begin{array}{l}F(4)=38.75, \\
p<.001, \\
R^{2}=.55\end{array}$ & $\begin{array}{l}F(4)=41.21, \\
p<.001, \\
R^{2}=.57\end{array}$ \\
\hline & $\begin{array}{l}\text { Grado } \\
\text { Escolar }\end{array}$ & $-.13 *$ & -.10 & -.05 & -.11 & $-.12 *$ \\
\hline & MT & $-.72 * * *$ & $-.60 * * *$ & $-.73 * * *$ & $-.68 * * *$ & $-.69 * * *$ \\
\hline & $\begin{array}{l}\text { RC-TEH } \\
\text { ME-TEH }\end{array}$ & $\begin{array}{l}-.02 \\
.11(p=.06)\end{array}$ & $\begin{array}{l}.01 \\
.09\end{array}$ & $\begin{array}{l}-.07 \\
.20 * *\end{array}$ & $\begin{array}{l}-.05 \\
.16^{*}\end{array}$ & $\begin{array}{l}-.04 \\
.14^{*}\end{array}$ \\
\hline
\end{tabular}

Notas $^{*} p<.05 ; * * p<.01 ; * * * p<.001$. En todos los casos se informa el $R^{2}$ corregido

\section{Discusión}

El objetivo de este trabajo fue analizar la capacidad explicativa de las estrategias de regulación de la emoción RC-TEH y ME-TEH sobre el DA, controlando el peso de las dif-MT en un grupo de niños que se encuentran en los últimos años de la escolaridad primaria. Los resultados mostraron que el ME-TEH se asoció con las dimensiones éxito (marginalmente) y productividad académica y las áreas de matemáticas y lectura, incluso cuando se introdujo difMT como variable de control. No se observaron asociaciones significativas de la RC-TEH con el DA, ni de ninguna de las estrategias sobre la dimensión control del impulso del DA.

Respecto de la asociación entre el ME-TEH y el DA, nuestros resultados son similares a los obtenidos por otros estudios. Xu et al. $(2015,2016)$ habían hallado relaciones del ME-TEH con el rendimiento en pruebas estandarizadas de matemáticas en adolescentes de 13 y 15 años de edad promedio. El ME-TEH se caracteriza por la capacidad de modificar los pensamientos sobre las propias habilidades para lograr impactar sobre la emoción $(\mathrm{Xu}, 2016)$. Es posible que frente a las emociones negativas que generan las tareas escolares, los niños que se consideran capaces de resolverlas y piensan que es importante mantener la calma, se encuentran en mejores condiciones para permanecer en ellas y completarlas. Si bien las asociaciones son relativamente bajas, resulta factible que estos niños sean percibidos por sus docentes con mayor éxito y productividad académica; es decir, con producciones de mayor calidad, así como con un repertorio de comportamientos que conducen a esa mayor calidad. Sobre la asociación con matemáticas y 
lectura, resulta posible que los niños que regulan sus emociones frente a las tareas de estas áreas específicas sean también percibidos por sus docentes con mayor dominio de las mismas.

Estas asociaciones entre el ME-TEH y diferentes dominios del DA, se hallaron incluso cuando se incorporó un predictor de ambas variables como dif-MT; lo que permite que el análisis de la capacidad predictiva del ME-TEH resulte más preciso. De hecho, en relación a la asociación entre dif-MT y DA, los resultados muestran que las dif-MT se asocian consistentemente con las diferentes dimensiones del DA. Esto está en línea con la evidencia que muestra que la memoria de trabajo cumple un rol clave en el DA. Si bien la medida de memoria de trabajo utilizada es una medida de informe, estudios recientes indican que la medición de las dif-MT mediante cuestionarios como el utilizado presenta asociaciones con tareas de DA (e.g. Vernucci et al., 2020). Si bien el mayor porcentaje explicativo de las dimensiones del DA estuvo dado por las dif-MT, el ME-TEH continuó teniendo poder predictivo, lo que estaría indicando que la regulación emocional posee una contribución única al DA más allá de otras variables teóricamente relacionadas como las funciones ejecutivas. En este sentido, nuestros resultados guardan similitud con los hallados por LaFavor (2012).

En relación a la ausencia de asociaciones significativas entre la RC-TEH y el DA, nuestros resultados son similares a los obtenidos por Xu et al. (2015, 2016), quienes obtuvieron asociaciones del ME-TEH con pruebas estandarizadas de matemática, pero no de la RC-TEH. Es posible que pensar en términos positivos sobre las propias capacidades para afrontar las tareas posea mejores beneficios que pensar en la situación en sí en términos más optimistas. En este sentido, el autoconcepto académico favorable -entendido como las percepciones respecto de uno mismo en el dominio escolar- guarda relaciones positivas con el DA (e.g. Peralta Sánchez \& Sánchez Roda, 2003). En relación a la RC-TEH, quizás la reinterpretación de la "obligación" de hacer las tareas como una oportunidad para el aprendizaje no posea un impacto en el grado de compromiso con la permanencia y tendencia a completarlas. El trabajo de Davis y Levine (2013) encontró que la implementación de la reevaluación cognitiva mejoró el recuerdo de material educativo en niños de escolaridad primaria. En este estudio, los niños fueron instruidos y entrenados para su implementación efectiva, por lo que podría suceder que la frecuencia autoinformada de su utilización espontánea no refleje asociaciones con el DA.

En referencia a la relación entre las estrategias y el control del impulso, esperábamos una asociación positiva, debido a que la capacidad de reinterpretar las propias habilidades y la situación en términos más positivos debería favorecer la permanencia en la tarea y la disminución de comportamientos como abandono de la misma, realización rápida y con poco detalle, etc. Sin embargo, no hemos encontrado una asociación. Es posible que simplemente otras variables, como las dif-MT y otras no contempladas en este trabajo, expliquen el control del impulso en situaciones académicas. Incluso podría suceder que otros procesos de regulación emocional tengan mayor poder predictivo y que las estrategias aquí contempladas resulten demasiado específicas.

Entre las limitaciones de este trabajo es posible mencionar, por un lado, la naturaleza transversal del diseño. Esto no permite descartar relaciones bidireccionales entre las variables. Sin embargo, otros estudios similares poseen modelos con la misma direccionalidad que el nuestro, lo que aporta mayor validez a los resultados obtenidos. Por otro lado, se debe mencionar también la naturaleza exclusiva de autoinforme de los instrumentos y la utilización de informante único (i.e. docentes) para evaluar dif-MT y DA. Esta metodología es vulnerable a los sesgos de respuesta y a la mayor varianza común entre las variables (véase Duckworth \& Yeager, 2015). No obstante, a partir de los 9 años de edad los niños son altamente capaces de informar sus propios estados emocionales (Rydell, Thorell \& Bohlin, 2007), y se ha indicado que los informes docentes proveen información amplia y representativa del DA, complementaria a las tareas de rendimiento (Graziano et al., 2007). Además, si bien las dif-Mt y el DA fue informado por los docentes, las estrategias de regulación emocional fueron reportadas por los propios niños, lo que 
aporta mayor validez a las asociaciones -si bien bajas- encontradas. En tercer lugar, la confiabilidad del instrumento para evaluar las estrategias de regulación emocional fue relativamente baja. Sin embargo, las respuestas dadas por los niños suelen ser más inestables que las ofrecidas por los adultos, por lo que correlaciones de .30 pueden considerarse con valor explicativo (e.g. Mischel, Zeiss \& Zeiss, 1974). Finalmente, el tamaño de la muestra es reducido y la selección de la misma fue intencional lo que limita las posibilidades de generalización de los resultados. No obstante, otros estudios en niños y adolescentes hallaron relaciones similares a las nuestras.

Futuros estudios podrían ganar precisión al realizar estudios longitudinales, con muestras más representativas y con la utilización de medidas de rendimiento y/o múltiples informantes. Especialmente, incorporar otras funciones ejecutivas como variables de control. Por ejemplo, la inhibición y la flexibilidad cognitiva han resultado predictores tanto del DA como de la regulación emocional (e.g. Aran-Filippetti \& López, 2014; Schmeichel \& Tang, 2013; 2015). La incorporación de otros procesos de regulación emocional permitiría conocer la contribución diferencial de cada uno sobre el DA, lo que ampliaría el conocimiento del rol de la regulación emocional en el aprendizaje.

\section{Conclusiones}

A pesar de las limitaciones, los resultados obtenidos representan una contribución al mostrar que la regulación de la emoción específica de tareas escolares para el hogar posee un rol en la explicación del DA, en una población infantil que ha sido escasamente estudiada. Especialmente, aquellos niños que se percibieron a sí mismos con mayor capacidad de ME-TEH fueron evaluados por sus docentes con mayor éxito y productividad académica, así como con mayores rendimientos en lectura y matemáticas. Incluso esta asociación se mantuvo cuando se controlaron funciones cognitivas fuertemente comprometidas en ambas variables, como las difMT. Completar las tareas escolares es un predictor importante del DA (Cooper et al., 2006), por lo que regular las emociones negativas que éstas provocan impacta positivamente sobre el mismo. En este sentido, estos resultados podrían constituir insumos para el contenido de programas de promoción de la regulación emocional diseñados para contextos escolares.

\section{Referencias}

Alloway, T.P. (2007). Automated working memory assessment. London: Pearson Assessment.

Alloway, T.P. \& Alloway, R.G. (2010). Investigating the predictive roles of working memory and IQ in academic attainment. Journal of Experimental Child Psychology, 106(1), 2029. doi: $10.1016 /$ j.jecp.2009.11.003

Alloway, T.P., Gathercole, S.E. \& Kirkwood, H.J. (2008). Working Memory Rating Scale. UK: Pearson Education.

American Psychological Association (2010). Ethical principles of psychologists and code of conduct. Washington, DC: Autor. Recuperado de http://www.apa.org/ethics/code/principles.pdf

Andrés, M. L., Stelzer, F., Juric, L. C., Introzzi, I., Rodríguez-Carvajal, R., \& Guzmán, J. I. N. (2017). Regulación emocional y desempeño académico: revisión sistemática de sus relaciones empíricas. Psicologia em Estudo, 22(3), 299-311

Andrés, M. L., Castañeiras, C., Stelzer, F., Canet Juric, L., \& Introzzi, I. (2016). Funciones Ejecutivas y Regulación de la Emoción: evidencia de su relación en niños. Psicología Desde El Caribe, 33(2), 169-189.

Arán-Filippetti, V. \& López, M.B. (2014) The Role of Executive Functions in Academic Competences: An Analytical Review. En K. Bennet (Ed.) Executive Functioning. Role in 
Early Learning Processes, Impairments in Neurological Disorders and Impact of Cognitive Behavior Therapy (CBT) (pp. 305-321). New York NY: Nova Science Publishers, Inc.

Bas, G., Senturk, C., \& Cigerci, F. M. (2017). Homework and academic achievement: A metaanalytic review of research. Issues in Educational Research, 27(1), 31-50.

Baddeley, A. (2012). Working memory: Theories, models and controversies. Annual Review of Psychology, 63, 1-29. doi: 10.1146/annurev-psych-120710-100422

Bennett, K. J., Brown, K. S., Boyle, M., Racine, Y. \& Offord, D. (2003). Does low reading achievement at school entry cause conduct problems? Social Science \& Medicine, 56, 2443-2448. doi: 10.1016/S0277-9536(02)00247-2

Bull, R. \& Lee, K. (2014). Executive functioning and mathematics achievement. Child Development Perspectives, 8, 36-41. doi: 10.1111/cdep.12059

Cain, K., Oakhill, J. \& Bryant, P. (2004). Children's reading comprehension ability: Concurrent prediction by working memory, verbal ability, and component skills. Journal of Educational Psychology, 96, 31-42. doi: 10.1037/0022-0663.96.1.31

Canet-Juric, L., Andrés, M. L., García-Coni, A., Richard's, M. M., \& Burín, D. (2017). Desempeño en memoria de trabajo e indicadores comportamentales: Relaciones entre medidas directas e indirectas. Interdisciplinaria. Revista de Psicología y Ciencias Afines, 34(2), 369-387. doi: 10.16888/I.V34I2.345

Center on the Developing Child at Harvard University (2011). Building the Brain's "Air Traffic Control" System: How Early Experiences Shape the Development of Executive Function: Working Paper No. 11. Recuperado de www.developingchild.harvard.edu.

Consejo Nacional de Investigaciones Científicas y Técnicas (2006). Lineamientos para el comportamiento ético en las ciencias sociales y humanidades (CSyH). Buenos Aires, Argentina: Ministerio de Educación, Ciencia y Tecnología. Recuperado de http://www.conicet.gov.ar/wp-content/uploads/RD-20061211-2857.pdf

Cooper, H., Robinson, J. C., \& Patall, E. A. (2006). Does Homework Improve Academic Achievement? A Synthesis of Research, 1987-2003. Review of Educational Research, 76(1), 1-62.

Davis, E. L. \& Levine, L. J. (2013). Emotion regulation strategies that promote learning: reappraisal enhances children's memory for educational information. Child Development, 84(1), 361-374. doi: 10.1111/j.1467-8624.2012.01836.x

Diamond, A. (2013). Executive Functions. Annual Review of Psychology, 64, 135-168. doi: 10.1146/annurev-psych-113011-143750

Duckworth, A. L., \& Yeager, D. S. (2015). Measurement matters: Assessing personal qualities other than cognitive ability for educational purposes. Educational Researcher, 44(4), 237-251. doi: 10.3102/0013189X15584327

DuPaul, G. J., Rapport, M. D., \& Perriello, L. M. (1991). Teacher ratings of academic skills: The development of the Academic Performance Rating Scale. School Psychology Review, 20(2), 284-300.

Graziano, P. A., Reavis, R. D., Keane, S. P., \& Calkins, S. D. (2007). The Role of Emotion Regulation and Children's Early Academic Success. Journal of School Psychology, 45(1), 3-19. doi: 10.1016/j.jsp.2006.09.002

Gross, J. J. (2002). Emotion regulation: Affective, cognitive, and social consequences. Psychophysiology, 39(3), 281-291. doi: 10.1017/S0048577201393198

Gross, J. J. (2014). Emotion Regulation: Conceptual and Empirical Foundations. En J. J. Gross (Ed.), Handbook of Emotion Regulation. Second Edition (Second). New York - London: The Guilford Press.

Gross, J. J., \& Thompson, R. A. (2007). Emotion regulation: Conceptual foundations. En J. J. Gross (Ed.), Handbook of emotion regulation (pp. 3-24). New York: Guilford Press. 
Hambleton, R. K. (2001). The next generation of the ITC test translation and adaptation guidelines. European Journal of Psychological Assessment, 17(3), 164-172. doi: 10.1027//1015-5759.17.3.164

Hattie, J. (2009). Visible learning. A synthesis of over 800 meta-analysis relating to achievement. Oxon: Routledge.

Hofmann, W., Schmeichel, B. J., \& Baddeley, A. D. (2012). Executive functions and selfregulation. Trends in Cognitive Sciences, 16(3), 174-180. doi: 10.1016/j.tics.2012.01.006

Hollingshead, A. B. (2011). Four factor index of social status. Yale Journal of Sociology, 8, 2-52.

Jamieson, J. P., Mendes, W. B., Blackstock, E., \& Schmader, T. (2010). Turning the knots in your stomach into bows: Reappraising arousal improves performance on the GRE. Journal of Experimental Social Psychology, 46(1), 208-212. doi: 10.1016/j.jesp.2009.08.015

Johns, M., Inzlicht, M., \& Schmader, T. (2008). Stereotype threat and executive resource depletion: examining the influence of emotion regulation. Journal of Experimental Psychology: General, 137(4), 691-705. doi: 10.1037/a0013834

LaFavor, T. L. (2012). The Impact of Executive Function and Emotional Control and Understanding on the Behavioral Functioning and Academic Achievement of Children Living in Emergency Homeless Shelters. University of Minnesota.

Leroy, V., Grégoire, J., Magen, E., Gross, J. J., \& Mikolajczak, M. (2012). Resisting the sirens of temptation while studying: Using reappraisal to increase focus, enthusiasm, and performance. Learning and Individual Differences, 22(2), 263-268. doi: 10.1016/j.lindif.2011.10.003

Maltese, A. V., Tai, R. H., \& Fan, X. (2012). When is homework worth the time? Evaluating the association between homework and achievement in high school science and math. The High School Journal, 96(1), 52-72. doi: 10.1353/hsj.2012.0015

Martin, R. E. \& Ochsner, K. N. (2016). The neuroscience of emotion regulation development: Implications for education. Current Opinion in Behavioral Sciences, 10, 142-148. doi: 10.1016/j.cobeha.2016.06.006

McRae, K., Jacobs, S. E., Ray, R. D., John, O. P. \& Gross, J. J. (2012). Individual differences in reappraisal ability: Links to reappraisal frequency, well-being, and cognitive control. Journal of Research in Personality, 46(1), 2-7. doi: 10.1016/j. jrp.2011.10.003

Mischel, W., Zeiss, R., \& Zeiss, A. (1974). Internal-external control and persistence: Validation and implications of the Stanford Preschool Internal-External Scale. Journal of Personality and Social Psychology, 29(2), 265-278. doi: 10.1037/h0036020

Montero, I., \& León, O. G. (2007). A guide for naming research studies in Psychology. International Journal of Clinical and Health Psychology, 7(3), 847-862.

Parsons, S. \& Bynner, J. (2005). Does numeracy matter more? London: National Research and Development Centre for Adult Literacy and Numeracy.

Pascual, L., Galperín, C. y Bornstein, M. (1993) La medición del nivel socioeconómico y la psicología evolutiva: el caso argentino. Revista Interamericana de Psicología, 27(1), 5974.

Pe, M. L., Koval, P., Houben, M., Erbas, Y., Champagne, D., \& Kuppens, P. (2015). Updating in Working Memory Predicts Greater Emotion Reactivity to and Facilitated Recovery from Negative Emotion-Eliciting Stimuli. Frontiers in Psychology, 6(372). doi: 10.3389/fpsyg.2015.00372

Pe, M. L., Raes, F. \& Kuppens, P. (2013). The cognitive building blocks of emotion regulation: Ability to update working memory moderates the efficacy of rumination and reappraisal on emotion. PLOS one, 8(7), 1-11. doi: 10.1371/ journal.pone.0069071 
Peralta Sánchez, F. J., \& Sánchez Roda, M. D. (2003). Relaciones entre el autoconcepto y el rendimiento académico, en alumnos de Educación Primaria. Electronic journal of research in educational psychology, 1(1).

Raghubar, K. P., Barnes, M. A. \& Hecht, S. A. (2010). Working memory and mathematics: A review of developmental, individual difference, and cognitive approaches. Learning and Individual Differences, 20, 110-122. doi: 10.1016/j.lindif.2009.10.005

Rydell, A. M.; Thorell, L. B. \& Bohlin, G. (2007). Emotion regulation and relation to social functioning: An investigation of child self-report. European Journal of Developemental Psychology, 1, 293-313. doi: 10.1080/17405620600783526

Sautú, R. (1989). Teoría y técnica en la medición del status ocupacional: escalas objetivas de prestigio (Documento de trabajo). Buenos Aires: UBA, Instituto de Ciencias Sociales.

Schmeichel, B. J., \& Tang, D. (2013). The relationship between individual differences in executive functioning and emotion regulation: A comprehensive review. En J. P. Forgas \& E. Harmon-Jones (Eds.). The control within: Motivation and its regulation (pp. 133152). New York: Psychology Press.

Schmeichel, B. J., \& Tang, D. (2015). Individual Differences in Executive Functioning and Their Relationship to Emotional Processes and Responses. Current Directions in Psychological Science, 24(2), 93-98. http://dx.doi.org/10.1177/0963721414555178

Steinmayr, R., Meibner, A., Weidinger, A., \& Wirthwein, 1. (2015). Academic Achievement. Oxford: UK: Oxford Bibliographies.

St Clair-Thompson, H. L., \& Gathercole, S. E. (2006). Executive functions and achievements in school: Shifting, updating, inhibition, and working memory. Quarterly Journal of Experimental Psychology, 59(4), 745-759. doi: 10.1080/17470210500162854

Tornimbeni, S., Pérez, E, Olaz, F., \& Fernández, A. (2004) Introducción a los Tests Psicológicos. ( $3^{\circ}$ Ed.). Córdoba, Argentina: Ed. Brujas.

Vernucci, S., Galli, J. I., Andrés, M. L., Zamora, E. V., Richards, M. M., Canet-Juric, L., \& Burin, D. I. (2020). Working Memory Screening in Latin American Children: Psychometric Properties of a Spanish Version of the Working Memory Rating Scale. Journal of Research in Childhood Education, 34, 463-475. doi: 10.1080/02568543.2019.1710730

Wechsler, D. (2004). Wechsler Scale of Intelligence IV. London: PearsonAssessment.

$\mathrm{Xu}$, J. (2016). Emotion regulation in mathematics homework: An empirical study. Journal of Educational Research, 111(1), 1-11. doi: 10.1080/00220671.2016.1175409

Xu, J., Fan, X., \& Du, J. (2015). Homework Emotion Regulation Scale: Psychometric Properties for Middle School Students. Journal of Psychoeducational Assessment, September, 1-11. doi: $10.1177 / 0734282915603542$

Xu, J., Fan, X., \& Du, J. (2016). Homework Emotion Regulation Scale: Confirming the Factor Structure With High School Students. Journal of Psychoeducational Assessment, September, 1-5. doi: 10.1177/0734282915603542

Contribución de los autores: a) Concepción y diseño del trabajo; b) Adquisición de datos; c) Análisis e interpretación de datos; d) Redacción del manuscrito; e) revisión crítica del manuscrito.

M.L.A. ha contribuido en a,c,d; S.V. en a,b,c,e ; A.G.C. en c,d,e; M.M.R. en c,d,e; M.L.A. en b,c,e; R.P. en b,c,e..

Editora científica responsable: Dra. Cecilia Cracco 